Revista de Comunicación y Salud, 2017, Vol. 7, pp. 119-149

Editado por Cátedra de Comunicación y salud.

ISSN: 2174-5323 (impreso) 2173-1675 (electrónico)

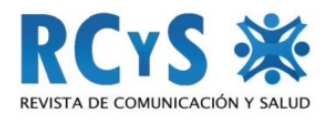

Enviado 27 de septiembre de 2017

Aprobado 7 de noviembre 2017

\title{
TECNO-ADICCIÓN AL SEXO EN LA POBLACIÓN JUVENIL: PROPUESTA DEL DISEÑO DE UNA ESCALA DE DETECCIÓN TEMPRANA
}

\section{TECHNO-ADDICTION to sex in the youth population: Proposal for the design of an early detection scale}

\author{
Olga Serrano Villalobos ${ }^{1}$, Victoria Cuesta Díaz* \\ Universidad Complutense de Madrid \\ *Hospital Quirón de Madrid
}

\section{RESUMEN}

Debido a una fuerte penetración de las TICs en la vida cotidiana de los más jóvenes, el comportamiento sexual de éstos se ve afectado por el uso de las TICs. Internet parece facilitar una exposición temprana entre los menores a contenidos pornográficos de adultos, mientras que el cerebro de los más jóvenes no estaría totalmente preparado para cierto tipo de imágenes sexuales. La comunidad científica ofrece escalas para medir este tipo de problemática, dirigidas, principalmente, a adultos. Ante la necesidad, se propone una herramienta adaptada a niños y adolescentes, capaz de medir sin hacer mención alguna a cualquier figura sexual explícita. El objetivo es presentar una revisión bibliográfica sobre las escalas previas que nos dirija hacia el análisis de las variables más relevantes a la hora de proponer una herramienta válida de cribado y detección temprana para la adicción al sexo por Internet en la población juvenil, así como plantear un nuevo modelo teórico para comprender el fenómeno de tecnoadicción al sexo vs. adicción al ciber-sexo. Para ello realizamos un meta-análisis bibliográfico de escalas existentes en la materia así como el previo estudio de otros modelos teóricos anteriores para poder construir en el futuro una nueva herramienta de detección temprana, basada en el análisis del comportamiento a través del estudio de las emociones como origen-causal del comportamiento, y capaz de informar si un joven, hombre o mujer, tiene o no problemas relacionados con la sexualidad e Internet, así como detectar que trastornos (adyacentes) forman parte de dicho problema.

Palabras clave: Escala de detección y cribado, Adicción, Sexo, Nuevas Técnologías de la Información y la Comunicación (TICs), Emociones, Jóvenes, Comportamientos, Clínica.

\footnotetext{
${ }^{1}$ Autor de contacto: Olga Serrano Villalobos olga.serrano@gmail.com
} 
Tecno-adicción al sexo en la población juvenil: propuesta del diseño de una escala de detección temprana

\begin{abstract}
Due to the strong penetration of ICT in the daily life of young people, sexual behavior has been affected by the use of ICT. The Internet seems to facilitate early exposure of minors to adult pornographic content, while the younger's brain is not fully prepared for the type of sexual imagery. The scientific community offers scales to measure this type of problem, directed, mainly, to adults. Given the need, it is proposed a tool adapted to children and adolescents capable of measuring without mentioning any explicit sexual figure. The objective is to present a bibliographic review on the previous scales that will guide us towards the analysis of the most relevant variables when proposing a valid tool for screening and early detection for Internet sex addiction in the youth population, as well as to propose a new theoretical model to understand the phenomenon of sexaddiction techno-addiction vs. addiction to cyber-sex. To do this we perform a bibliographic meta-analysis of existing scales in the subject matter as well as the previous study of other previous theoretical models to be able to construct in the future to new tool of early detection, based on the analysis of the behavior through the study of the emotions as causal-origin of behavior, and able to inform whether a young man or woman has or does not have problems related to sexuality and the Internet, as well as to detect that (adjacent) disorders are part of this problem .

Keywords: Screening and Screening Scale, Addiction, Sex, New Information and Communication Technologies (ICTs), Emotions, Youth, Behavior, Clinic.

\section{Para citar el artículo}

Serrano Villalobos, O., Cuesta Díaz, V. (2017). Tecno-adicción al sexo en la población juvenil: propuesta del diseño de una escala de detección temprana. Revista de Comunicación y Salud, vol. 7, 119-149. Recuperado de http://revistadecomunicacionysalud.org/index.php/rcys/article/view/130
\end{abstract}

\title{
1. INTRODUCCIÓN
}

Como introducción a las teorías y modelos relevantes del sexo en Internet, en primer lugar, es importante considerar que las dos formas de adicción a Internet con contenido sexual son el cibersexo (interacción) y la pornografía (no interacción) (Silvia Sánchez Zaldívar, Itziar Iruarrizaga Díez, 2009).

La pornografía en Internet se considera una actividad sin interacción, donde el objetivo principalmente es la "excitación sexual" con o sin masturbación final. Parece que este tipo de actuación resulta más íntima que la ofrecida en el cibersexo, ya que no existe interacción alguna, y el individuo se encuentra viendo o contemplando fotografías o vídeos con contenido sexual en solitario principalmente, por lo que el desarrollo de pensamientos, sentimientos, y creencias en ese momento se ve fortalecidos a través de las fantasías, siendo considerado un momento de intimidad sexual en solitario. En esta parte, quizá la búsqueda de "poder sexual" se encuentre más delimitada, ya que no hay que pedir permiso.

En este sentido, tanto la pornografía como el cibersexo, coinciden en que el individuo busca la excitación sexual en primera instancia, seguido o no de la masturbación. De 
Tecno-adicción al sexo en la población juvenil: propuesta del diseño de una escala de detección temprana

esta manera podemos pensar que el elemento "excitación sexual" podría ser la base para entender el comportamiento del sujeto. Además, de esta manera también, es posible decir que en la búsqueda de excitación sexual lo que realmente se trata es de encontrar emociones, por lo que la implicación de emociones parece evidente, entendiendo que éstas vendrían dadas tanto en la interiorización como en la exteriorización de las mismas.

A partir del uso de Internet para el sexo surge el vocablo "cibersexo", para definir una forma de sexo virtual por el cual dos o más personas se envían mensajes sexualmente explícitos con el objetivo de estimular deseos sexuales como también fantasías sexuales, ya que la imaginación juega un papel importante en estos procesos, puesto que en el cibersexo se juega y se crean personajes y personalidades distintas con motivo de realizar un juego erótico. El fin último de esta práctica, es la masturbación o conseguir citas para relaciones sexuales reales. La particularidad del sexo virtual es que se trata de una forma de sexo sin contacto físico, lo que evita enfermedades de trasmisión sexual y embarazos, que además triunfa por su anonimato (Silvia Sánchez Zaldívar, Itziar Iruarrizaga Díez, 2009).

Lo cierto es que con la llegada de Internet se introduce un nuevo elemento no existente anteriormente que es la interactividad, y ello, parece resultar un éxito entre los usuarios, ya que proporciona "nuevas formas de excitación sexual" a partir de las nuevas prácticas. Además, el sexo virtual pone a prueba al usuario, al tener que realizar un seguimiento del el objeto deseado y obtener su confirmación. En esta posición también se observa un mayor "poder sexual", así como se percibe también una cierta competitividad sexual.

El cibersexo ocurre a través del chat o mensajería instantánea, correo electrónico, juegos, redes sociales, etc., que puede hacerse visual gracias a las cámaras incorporadas en los ordenadores, Smartphones, tablets, etc., además, el cibersexo también puede darse en Smartphones y teléfonos móviles a través de SMS y de llamadas telefónicas incluida la versión de teléfono erótico de pago. Se observa una nueva condición que no es otra que la interactividad principalmente con contenidos audiovisuales.

Estas nuevas prácticas fruto del avance tecnológico y cuyo encuentro es el sexo, favorecen la distancia, y todo ello puede ocurrir a pesar que los participantes estén en diferentes continentes.

Internet se mueve en la ambigüedad entre los beneficios y lo inconvenientes, y al referirse al sexo, es posible que se necesite un control adecuado y eficaz debido a la existencia entre una relación directa entre cibersexo y pedofilia, que prolifera debido a las características particulares de Internet y de las nuevas tecnologías de la Información y Comunicación. Es obvio que el sexo no es nada nuevo y el uso de la pornografía tampoco. "En el siglo XIX las preocupaciones se centraban en los riesgos para la salud, como el modelo de la enfermedad de la masturbación. Sin embargo, a partir de segunda mitad del siglo XX la preocupación eran los daños sociales que van desde la objetivación y la degradación de las mujeres hasta la violencia sexual" (Aleksandar Stulhofer, Vesna Busko, Ivan Landripet, 2010), y la pedofilia. La pornografía infantil se convierte en un hecho.

Con respecto a los contenidos sexuales parece ser que con el tiempo han tenido una tendencia a la brutalidad por lo que en lo referente al sexo cualquier cosa es válida. El 
Tecno-adicción al sexo en la población juvenil: propuesta del diseño de una escala de detección temprana

recorrido histórico ha permitido ver la dureza o ante-sala de los contenidos actuales. Adicionalmente, la historia puede explicar cómo han ido cambiando los conceptos hasta la interiorización que se hace actualmente de la pornografía, y en consecuencia posibilita reflejar la actividad sexual que se lleva a cabo en la actualidad así como las herramientas que se utilizan para llegar a ella.

En la actualidad, la actividad sexual por Internet principalmente se relaciona con fotografías, vídeos o chats sexuales. Por lo que las actividades relacionadas son valoradas desde este trabajo por la visualización de pornografía (Conducta voyeurista), el envío/recepción de imágenes sexuales (comportamiento exhibicionista), conversaciones en chats o video-conferencia (fantasías). El fin último de este tipo de conexiones es la consecución de placer sexual exista o no masturbación final, aunque parece ser que la masturbación es la acción perseguida en la búsqueda última de placer sexual (masturbación compulsiva). Este estudio considera al referirse "buscar sexo por Internet" a buscar principalmente fotografías o vídeos eróticos o chats de sexo. Parece desde esta perspectiva que las actividades sexuales posibles en Internet se dividen en interactivas o no, y que la pornografía se diferencias de otro tipo de actividades sexuales posible en la red, como las redes sociales para conseguir relaciones sexuales, u otros. Con la llegada especialmente de las redes sociales para las relaciones amorosas o sexuales, los chats multimedia y la mensajería instantánea por Internet, los protagonistas de este tipo de fotos o video eróticos que antes eran profesionales, serán ahora los propios "usuarios" de Internet, por lo que la distribución de imágenes sexualmente explicitas cambia tomando mayor importancia el "usuario" como actor pornográfico. Ahora, cualquiera puede enviar fotos propias con el objetivo de estimular sexualmente a otra persona, en este caso "usuario". Además también se observan otras diferencias como el hecho que antes de la llegada de Internet la pornografía estaba centrada sobre todo en mostrar imágenes de mujeres, pero ahora con la masiva distribución de imágenes sexuales de "usuarios", el hombre también comienza a mostrar imágenes sugerentes ampliándose por tanto también la distribución de imágenes masculinas, ya sean dirigidas para mujeres o para otros hombres. Quizá sea en este nuevo acontecimiento donde se pueden generar confusiones de identidad sexual, así como ampliar espectros sexuales, y surgir algunos problemas relacionados con la sexualidad e Internet especialmente en los más jóvenes (niños y adolescentes), a partir de la interiorización y exteriorización de las emociones de éstos.

\section{OBJETIVOS}

Debido a que hay pocos cuestionarios que están centrados específicamente en la actividad sexual en Internet y en los problemas sexuales en Internet (Stefano Eleuteri, Francesca Tripodi, Irene Petruccelli, Roberta Rossi, Chiara Simonelli, 2014) derivados del uso de la tecnología con fines sexuales, este trabajo se centra en la creación de una herramienta que sea capaz de evaluar el origen causal de los comportamientos sexuales a través de Internet y su relación con la falta de control de impulsos y la perturbación en la vida diaria. Esta escala está acompañada de un modelo teórico que explica la evolución del fenómeno a través de las diferentes relaciones existentes en otros modelos previos, también a través de la extracción y análisis de factores integrantes, y a través de la reflexión de las diversas escalas existentes relacionadas a 
Tecno-adicción al sexo en la población juvenil: propuesta del diseño de una escala de detección temprana

través de los factores con el fenómeno.

Asimismo, entendemos la necesidad de crear una herramienta que sea específica para la población más joven, pudiéndose ser administrada también en niños y adolescentes, puesto que las herramientas previas encontradas principalmente están dirigidas a la población adulta.

El objetivo de este artículo es presentar una revisión bibliográfica sobre las escalas previas existentes que nos dirija en el futuro hacia la construcción de una herramienta válida de detección temprana para la adicción al sexo por Internet con varias metas:

En primer lugar, para poder obtener información sexual en la clínica y para poder detectar casos problemáticos con el uso de la tecnología en los más jóvenes a favor de la prevención de la salud.

Adicionalmente, se pretende realizar una nueva propuesta que englobe el término de adicción al sexo por Internet, incluyendo las dos posibles formas de adicción al sexo por Internet, como son el ciber-sexo y la pornografía (Silvia Sánchez Zaldivar et al.,2009), bajo el constructo de "Tecno-Adicción al sexo", pretendiendo de esta manera comprender el fenómeno de tecno-adicción al sexo vs. adicción al ciber-sexo.

En definitiva, lo que plantea este artículo es la posibilidad de construir un modelo fiable y rápido que permita medir personalidades que ofrezcan la posibilidad de observar el origen del comportamiento cuando un individuo acude a Internet a buscar sexo, así como una selección de herramientas previas que en el futuro nos impulsen a la creación de una escala de cribado y detección temprana adaptada para la población más joven.

\section{METODOLOGÍA}

El método que se sigue es realizar una revisión bibliográfica previa de los más relevantes y diferentes modelos teóricos existentes sobre el sexo por Internet.

Asimismo, se realiza un meta-análisis de las escalas existentes reconocidas en el actual DSM-5 como trastornos con el fin de poder justificar y delimitar este tipo de problemática y se valore como posible trastorno a partir del uso tecnológico. En otras palabras, se trata de ver la posibilidad a favor de la inclusión en la próxima versión del DSM de este tipo de patologías, ya que se pueden observar ciertas particularidades que sólo ocurren a través del uso tecnológico o mundo virtual. Es por ello, que en primera instancia, recurrimos a los trastornos que aparecen en el DSM-5, y en base a estos trastornos y a los modelos teóricos previos sea posible el planteamiento de una nueva herramienta de detección y cribado en el futuro.

En primer lugar, vamos a observar las grandes áreas o líneas de investigación principales que definen el fenómeno de adicción al sexo por Internet, de las que se extraerán posteriormente una selección de escalas que nos ayuden a la construcción en un futuro de la escala que se quiere proponer. Asimismo, se expondrá el modelo teórico que sostiene la escala que se propondrá en un futuro con respecto a las grandes áreas consideradas hasta ahora.

\subsection{Grandes áreas o líneas generales que dibujan los modelos que sostienen las escalas previas.}

Existen principalmente cinco grandes líneas de investigación con respecto a la 
Tecno-adicción al sexo en la población juvenil: propuesta del diseño de una escala de detección temprana

adicción al sexo por Internet sobre las que se halla esta investigación. Quizá es posible pensar que las líneas de investigación se han dividido desde un punto de vista general en función de los trastornos y los síntomas, principalmente. Estas grandes áreas definen el fenómeno a través de diferentes perspectivas, que son las siguientes:

1) La adicción al sexo por Internet se considera un subtipo de la adicción a Internet (Daniel L. King, Paul H. Delfabbro, Mark D. Griffiths, y Michael Gradisar, 2012; Block, 2008). Esta línea propone que la adicción tecnológica predomina sobre la adicción sexual. Se supone, en este sentido, que se defiende la idea por la que un individuo es adicto a Internet a través de diferentes aplicaciones o actividades entre las que se encuentra también la actividad sexual. El uso problemático de Internet se ha referido a través del uso excesivo principalmente de las actividades o funciones específicas de Internet como el correo electrónico, la pornografía, las compras, o los juegos de azar; y donde también se ha considerado la pérdida de tiempo online (Daniel L. King, Paul H. Delfabbro, Mark D. Griffiths, y Michael Gradisar, 2012). Así, un adicto a Internet, puede serlo a las compras, al sexo, a la navegación, al juego, a las redes sociales, a los chats, etc., en función de las actividades, o a todo a la vez. En definitiva, lo que determina el problema es el uso tecnológico. También se ha considerado la adicción a Internet, como plataforma tecnológica. Y además en esta categoría se incluye la adicción al dispositivo, por lo que se encuentran definiciones de adicción que se interesan por el teléfono móvil, ordenadores, Smartphones, etc. Esta área se ha interesado por la compulsividad tanto en los procesos de adicción a Internet, como en los procesos de adicción sexual por Internet como subtipo, así como en el uso de los diferentes dispositivos.

2) La adicción al sexo por Internet se considera un subtipo de la adicción al sexo. Está línea defiende que la adicción es sexual a través del uso de la tecnología; es decir, que se trata de una adicción sexual con un condicionante tecnológico. En este sentido, parece ser que Internet se utiliza como un medio para lograr la estimulación/excitación, para lograr gratificación/orgasmo, y ello puede afectar a la vida del individuo (Silvia Sánchez Zaldívar, Itziar Iruarrizaga Díez, 2009). Algunos autores, incluso definen a la adicción a la pornografía por Internet como un subconjunto del comportamiento hipersexual (Joshua B. Grubbs, Fred Volk, Julie J. Exline, Kenneth I. Pargament, 2015; Egan y Parmar, 2013; Kafka, 2009; Kaplan y krueger, 2010). Además, observamos que Internet es una fuente de diversidad sexual con contenidos sexuales bien diferenciados y variados, que corresponden a un cambio constante de éstos que invitan a los "usuarios" a no perderse nada nuevo, y esto puede potenciar un trastorno existente o no de orden sexual ya fuera por hipersexualidad, por parafilias, o por ambas. En este sentido, se observa que los métodos utilizados mediadores responsables del éxito, por un lado; y de la retroalimentación, por otro, no son otros que la llamada clásica de "novedad!!" y "gratis!!". Además, es posible comprender que estos nuevos trastornos sexuales que se forman a partir del uso tecnológico y que son definidos por sus propios elementos y características, tengan como base el trastorno sexual ya que el fin es la consecución de placer a través de la excitación o el orgasmo. De esta manera es posible definir un trastorno sexual 
Tecno-adicción al sexo en la población juvenil: propuesta del diseño de una escala de detección temprana

tecnológico exhibicionista, un trastorno sexual tecnológico voyeurista, o cualquier otro tipo de trastorno compatible con el uso tecnológico, principalmente de Internet. Además, también se observa un gasto sexual, como ocurre con otras adicciones, traducido por los esfuerzos que pone el individuo en satisfacer su adicción al sexo y por las consecuencias de un exceso.

3) La adicción al sexo por Internet se considera a partir de los síntomas. En esta línea de investigación que trabaja sobre los síntomas se ha observado que los síntomas se han considerado de distinta manera. Así nos encontramos con síntomas derivados del uso tecnológico, principalmente de Internet o dispositivos, y con síntomas derivados de los trastornos sexuales resultantes del uso de Internet para actividades sexuales; también con síntomas de angustia psicológica, y con síntomas psicológicos como la depresión, estrés, u otros, e incluso con síntomas psicológicos, psicosociales y psiquiátricos, como propone el modelo de este artículo. En este sentido, existen trabajos que apoyan estas premisas como el trabajo de Federico Tonioni, Lucio D'Alessandris, Carlo Lai, David Martinelli, Stefano Corvino, Massimo Vasale, Fabrizio Fanella Paola Aceto, Pietro Bria (2011) que relacionan la adicción a Internet con síntomas psicológicos como la ansiedad y la depresión, y donde éstos podrían detectar pacientes con trastorno de adicción a Internet; y también el trabajo de Chih-Hung Ko, Ju- Yu Yen, Cheng-Sheng Chen, Yi-Chun Yeh, Cheng-Fang Yen (2009) que relaciona la adicción a Internet en adolescentes con síntomas psiquiátricos como el déficit de atención e hiperactividad, la hostilidad, la depresión, la fobia social; proponiendo que dichos síntomas deben ser detectados como método de prevención. ChihHung Ko, Ju-Yu Yen, Shu-Chun Liu, Chi-Fen Huang y Cheng-Fang Yen (2008) ya anteriormente han asociado las conductas agresivas y la adicción a Internet y las actividades online en la adolescencia. Se observa una doble posibilidad que sirve para justificar el efecto de retroalimentación a través de pensar que el fenómeno se puede ver desde la existencia de un problema previo psicológico o psiquiátrico que lleve al individuo a un comportamiento problemático en Internet, pero además el mismo fenómeno se puede ver desde la perspectiva por la que el comportamiento problemático en Internet al individuo le reporta problemas psicológicos o psiquiátricos. Adicionalmente y con respecto a los síntomas, la adicción a Internet ha sido definida también por la imposibilidad para controlar el involucramiento y tendrá "características sintomatológicas" (que notablemente se diferencian de los estados normales de involucramiento) y "características disfuncionales" (y será a partir de estas disfuncionalidades o consecuencias la evaluación como entidad problemática) como han manifestado Nelly LamFigueroa, Hans Contreras-Pulache, Elizabeth Mori-Quispe, Martín NizamaValladolid, César Gutiérrez, Williams Hinostroza-Camposano, Erasmo Torrejón Reyes, Richard Hinostroza-Camposano, Elizabeth Coaquira-Condori, Willy David Hinostroza-Camposano, (2011) para la población adolescente, que a través de dos dimensiones evalúa: (1) la relación entre sintomatología de la Adicción a Internet y el tiempo de uso semanal, sexo o género, antecedentes de problemas de indisciplina y el plan de futuro, y (2) la relación entre la disfuncionalidad con antecedentes de problemas de indisciplina, plan de futuro y faltar sin motivo justificado a clase. Por último, observamos que los síntomas también pueden ser 
Tecno-adicción al sexo en la población juvenil: propuesta del diseño de una escala de detección temprana

analizados desde su manifestación interna y su manifestación externa; y en este sentido, el trabajo que aquí se propone se ha guiado por la interiorización y exteriorización desde el punto de vista de las emociones, sentimientos, pensamientos y creencias acompañado por la acción como comportamiento y actitud.

4) La adicción al sexo por Internet también se considera a partir de los criterios diagnósticos del DSM realizándose adaptaciones para todas las grandes áreas o líneas descritas anteriormente. En este sentido, se ha considerado principalmente el aumento de la preocupación por Internet, pasar cada vez más tiempo en Internet, los intentos fallidos para limitar el uso de Internet, los síntomas de abstinencia cuando se reduce el uso del Internet, utilizar Internet como un escape, el abandono de las tareas u obligaciones para pasar más tiempo en Internet, y el bajo rendimiento escolar como consecuencia de utilizar Internet, (Daniel L. King, Paul H. Delfabbro, Mark D. Griffiths, y Michael Gradisar, 2012), y sentimientos de culpa, entre otros. Estos criterios diagnósticos del DSM se han intentado adaptar a la visión de considerar el fenómeno como un subtipo de la adicción a Internet, como un subtipo a una adicción al sexo, y como sintomatología al ser adaptados los criterios diagnósticos como síntomas.

5) Y por último, la adicción al sexo por Internet se evalúa a partir del tratamiento, como se ha visto anteriormente. Dicho trabajo ha sido desarrollado de una manera más amplia desde la medicina, al poder conseguir más fácilmente tener un acceso patológico del fenómeno. En este sentido, se destaca la utilización de los criterios CONSORT que evalúan el tratamiento de adicción a Internet (Daniel L. King, Paul H. Delfabbro, Mark D. Griffiths, Michael Gradisar, 2011). Esta forma de evaluación hace posible pensar en la existencia de una estrecha relación entre síntomas y tratamiento para la evaluación de un trastorno, tal y como ocurre con las valoraciones de otras enfermedades comunes.

En todas estas áreas se ha hecho referencia de algún modo a trastorno del control de los impulsos. Este trabajo también se relaciona a la compulsividad, pero en este caso apoyada en factores psicológicos, psicosociales y psiquiátricos, que puedan llegar a reforzar la premisa de compulsividad, tanto para el modelo teórico como para la construcción de la escala, pretendiendo incluso que el cuestionario creado sea capaz de evaluar también sin ella. Siendo identificados, además, dichos factores con el origen del trastorno, y como componentes principales de esta investigación.

\subsection{Extracción de factores}

Según lo anterior, y a través del análisis de los modelos teóricos previos obtenemos los siguientes factores, considerados trastornos, que nos impulsan al análisis de las escalas existentes y reconocidas en el DSM-5. Dichos factores-trastornos son los siguientes:

\section{- La DEPRESIÓN}

- La ANSIEDAD 
Tecno-adicción al sexo en la población juvenil: propuesta del diseño de una escala de detección temprana

- La AGRESIVIDAD

- La FOBIA social

- EI DÉFICT DE ATENCIÓN E HIPERACTIVIDAD

- La COMPULSIVIDAD

- La OBSESIÓN

- La IMPULSIVIDAD

Además, de considerar estos factores como óptimos también, de esta forma, se obtienen factores independientes entre sí, que nos informan de la existencia a través de la relación directa entre los trastornos y el uso tecnológico, concretamente con fines sexuales. Asimismo, este planteamiento de considerar los factores independientes nos permitirán confirmar la existencia de comorbilidad para este tipo de trastornos así como indicar el trastorno en origen.

De una forma resumida, vamos a indicar los principales puntos que forman el modelo propuesto. En otras palabras, el modelo que se propone y del que se extraen los factores que lo componen está basado principalmente en:

1. El los trastornos reconocidos en el DSM-5, que componen nuestros factores, anteriormente citados: Depresión. Ansiedad, Agresividad, Fobia Social, Déficit de atención e Hiperactividad, Compulsiviad, Obsesión, Impulsividad.

2. El modelo se basa en el ORIGEN-CAUSAL del comportamiento, la COMPULSIVIDAD, y las características clásicas de la adicción (Tolerancia, Abstinencia, Perturbación de la vida diaria, y Recaída).

3. También está basado en que el origen del comportamiento se determina a partir de teorías de INTERIORIZACIÓN y EXTERIORZACIÓN (Sardinero García et al., 1997) de las emociones, siendo estás valoradas a partir de PENSAMIENTOS (Yo pienso), SENTIMIENTOS (Yo siento) y de CREENCIAS (Yo creo) como elementos de interiorización de las emociones y del COMPORTAMIENTO/ACCIÓN ejecutada (Yo actúo) como elemento de exteriorización de las emociones internas.

4. El modelo adicionalmente está basado en la COMORBILIDAD de los trastornos, siendo capaz de ser explicada a través de los trastornos adyacentes al ser considerados los factores como independientes.

5. El modelo se basa en la RETROALIMETACIÓN MÚLTIPLE, ya que considera la retroalimentación que ocurre en los procesos psicológicos (como el individuo se siente emocionalmente mal acude a Internet para sentirse mejor, así como el sujeto pasa tiempo enganchado a Internet con fines sexuales se siente mal después de las conexiones, donde en conjunto, unas emociones llevan a otras). Pero además también existe una retroalimentación en el propio proceso y diseño tecnológico de Internet, donde los contenidos son rápidos, cambiantes, múltiples, baratos, también a través de notificaciones y alertas, etc., (por lo que unos estímulos responden a unas emociones determinadas).

6. El modelo explicar el uso recreativo, y el aburrimiento considerando la CREACIÓN DE HÁBITO. 
Tecno-adicción al sexo en la población juvenil: propuesta del diseño de una escala de detección temprana

7. También las variables para el modelo que aquí se propone sugieren que los trastornos de adicción al sexo por Internet se consideran un subtipo de la adicción al sexo. También, se considera que los trastornos hipersexuales son un subtipo del trastorno obsesivo-compulsivo (TOC), debido a la relación directa entre hipersexualidad y compulsividad, una falta de control de impulsos y un pensamiento obsesivo recurrente.

8. El modelo que se propone incluye las dos formas posibles de adicción al sexo por Internet: Cibersexo y Pornografía que propone Silvia Sánchez Zaldivar et al. (2009).

9. Por último, el modelo que se propone desarrolla una teoría adicional donde los actuales trastornos sexuales parafílicos con el uso de la tecnología pasarían a ser considerados de la siguiente manera:

- Conducta Voyeurista $\rightarrow$ Conducta Tecno-Voyeurista

- Exhibicionismo $\rightarrow$ Tecno-Exhibicionismo

- Masturbación compulsiva $\rightarrow$ Tecno-Masturbación

Construyendo a parir de estos conceptos también el término "TECNO-ADICCIÓN", que aparece en el título de este artículo.

En resumen, el término TECNO-ADICCIÓN, por un lado, nace del intento de incluir todas las formas de actividad sexual en la red (cibersexo como activo-interactivo y pornografía como pasivo-no interactivo), y por otro, de tratar de definir las posibles parafílias que surgen como un nuevo fenómeno a partir del uso tecnológico.

\section{RESULTADOS Y DISCUSIÓN}

1) En primer lugar, sobre las grandes áreas que definen el fenómeno, la propuesta que se determina en este artículo, y que lo consideramos como parte del resultado que obtenemos se basa en lo siguiente:

La propuesta que se hace en este trabajo se encuentra a camino entre considerar, por un lado, la problemática a partir de un trastorno de la sexualidad que necesita el uso tecnológico para su consecución donde la parte tecnológica también favorece que se impulse la retroalimentación haciendo la tarea muy fácil, en cualquier momento y en cualquier lugar, y la parte sexual impulsa al individuo al uso de la tecnología para fines sexuales, en parte por el aprendizaje de lo beneficios tecnológicos de rapidez, comodidad, etc. Además, esta retroalimentación también ocurre entre los factores psicológicos descritos como trastornos por el DSM-5 como: la angustia, la tristeza, la autoestima, la agresividad, el déficit de atención e hiperactividad, comportamiento social, la compulsividad, y comportamiento obsesivocompulsivo, principalmente. Esto hace que coincida a través de los síntomas con la tercera área de investigación. Por tanto, es posible definir que este modelo es el resultado de considerar al fenómeno como un trastorno sexual en primera instancia y no tecnológico, definido a través de síntomas clínicos derivados del DSM-5, por lo que en este trabajo también se han considerado los criterios diagnósticos del DSM actual. Precisamente el DSM ha servido de guía para la definición de trastornos, la definición de comorbilidad y la definición de retroalimentación, a través de la 
Tecno-adicción al sexo en la población juvenil: propuesta del diseño de una escala de detección temprana

selección y extracción de los factores que en un futuro van a componer la escala que se propondrá más adelante. Además, este análisis que se hace también parte de una base por la que se interiorizan y se exteriorizan las emociones a partir de esos sentimientos, pensamientos, y creencias, al igual que ocurre con las conductas. En este sentido, se sigue el modelo de Sardinero García et al. (1997) cuando explica la "internalización" y "externalización" sobre el Cuestionario de Comportamiento Infantil de Achenbach -Child Behavior Checklist -(CBCL).

Por último, este trabajo considera que los trastornos de adicción al sexo por Internet se consideran un subtipo de la adicción al sexo. Además, se considera que los trastornos hipersexuales son un subtipo del trastorno obsesivo-compulsivo (TOC), y esto es debido a la relación directa entre hipersexualidad y compulsividad, una falta de control de impulsos y un pensamiento obsesivo recurrente.

2) En segundo lugar, como resultado obtenemos una selección de escalas previas que permitan en un futuro crear una posible herramienta válida de detección y cribado para uso clínico, entre otros, sobre la adicción al sexo por Internet. Por tanto, a continuación vamos a exponer las principales herramientas anteriores relevantes o escalas precedentes que nos conducirán en la creación de la futura herramienta o escala.

En un primer momento nos encontramos con unas escalas previas construidas que hacen referencia a la adicción al cibersexo y otras a la pornografía o a la exposición de material sexualmente explícito, como herramientas válidas para detectar una posible adicción al sexo por Internet. Dichas escalas están basadas principalmente en la compulsividad, la obsesión, en criterios generales de la adicción, la percepción de la gravedad, la culpa y angustia asociada (al uso), el gasto sexual, efectos negativos, la intención de uso, la excitación y masturbación, síntomas, y en la angustia psicológica o angustia emocional.

Además, algunas de estas escalas has sido construidas desde otros tests que medían la adicción a Internet, es decir, desde versiones modificadas de escalas previas de adicción a Internet.

\subsection{Herramientas anteriores relevantes. Las escalas precedentes}

Los estudios actuales han recopilado un variado espectro de datos a través de entrevistas, cuestionarios, observaciones, análisis de contenido y registro de archivos de registro de Internet, siendo las encuestas estandarizadas por escrito el método más común de intervención, además de los cuestionarios ad hoc (Stefano Eleuteri, Francesca Tripodi, Irene Petruccelli, Roberta Rossi, Chiara Simonelli, 2014). Parece ser que algunos de los mayores inconvenientes que se han tenido a este respecto es que la mayoría de estos estudios se han realizado a través de cuestionarios y datos publicados en sitios web en donde no se han evaluado las propiedades psicométricas (Stefano Eleuteri, Francesca Tripodi, Irene Petruccelli, Roberta Rossi, Chiara Simonelli, 2014; Albright, 2008; Boies, Cooper y Osborne, 2004; Cooper, Griffin-Shelley, Delmonico y Mathy, 2001; Cooper, Månnson, Deneback, Tikkanen, y Ross, 2003; Cooper, Scherer, y Mathy, 2004; Corley y Hook, 2012; Daneback, Cooper, y Månsson, 
Tecno-adicción al sexo en la población juvenil: propuesta del diseño de una escala de detección temprana

2005; Daneback, Månsson, y Ross, 2007; Grov, Gillespie, Royce, y Lever, 2011; Ross, Daneback , Månsson, Tikkanen, y Cooper, 2003; Ross, Månsson, y Daneback, 2012).

Las evaluaciones que se han realizado del fenómeno han sido desde psicológicas, (incluyendo historias sexuales pasadas, examen del estado mental y evaluaciones de trastornos comórbidos) hasta encuestas heurísticas simples online (Joshua B. Grubbs, John Sessoms, Dana M. Wheeler, y Fred Volk, 2010; Cooper et al., 1999; Delmonico y Griffin, 2008; Putnam, 2000; Putnam y Maheu, 2000).

Entre las herramientas específicas empleadas para evaluar los problemas generados a partir de un uso de Internet para la actividad sexual se destacan según Stefano Eleuteri, Francesca Tripodi, Irene Petruccelli, Roberta Rossi, Chiara Simonelli, (2014) las siguientes escalas:

- La prueba de Detección de Sexo en Internet -Internet Sex Screening Test(ISST), de Delmonico (1997), consta de 25 ítems falsos que clasifican a los sujetos en tres categorías: bajo riesgo (1-8), en riesgo (9-18), y alto riesgo (>19). Esta herramienta evalúa siete factores: (1) La Compulsividad sexual online -una medida de problemas sexuales online, (2) el Comportamiento sexual online -una medida de la tendencia a participar en interacciones interpersonales con otros durante el comportamiento sexual online como salas de chats sexuales, (3) el Comportamiento sexual online aislado -tendencia a participar en el comportamiento sexual online en solitario como ver pornografía, (4) el Gasto sexual online -tendencia a comprar material sexual y/o unirse a grupos o sitios web relacionados con el sexo a través de Internet, (5) el Interés en el comportamiento sexual -tendencia a usar el ordenador para actividades sexuales, por ejemplo, marcar los sitios sexuales. (6) También mide la tendencia a tener acceso a sitios sexuales de ordenadores distintos del ordenador de casa, y (7) la tendencia a ver material sexual ilegal en Internet. Existen otras versiones posteriores como el Internet Sex Screening Test (ISST) de Delmonico y Miller (2003) que apoya la existencia de tres componentes básicos en el comportamiento adictivo que implica: (1) Una incapacidad para detener el comportamiento, (2) efectos negativos significativos debido a la conducta, y (3) una obsesión generalizada con el comportamiento. EI ISST se ha considerado un instrumento prometedor para evaluar comportamientos compulsivos y adictivos relacionados con la pornografía en Internet (Joshua B. Grubbs, John Sessoms, Dana M. Wheeler, y Fred Volk, 2010), principalmente para adultos, por lo que los niños y adolescentes no estarían considerados para esta herramienta. Otros instrumentos diferentes han sido creados a partir de elementos que se han tomado del ISST para su análisis y evaluación. En España, Rafael Ballester Arnal, Ma Dolores Gil Llario, Sandra Gómez Martínez y Beatriz Gil Juliá (2010) han adaptado y validado el ISST para su administración en la población española donde se han mostrado cinco factores: (1) Compulsividad sexual online (como componente más patológico), (2) conducta sexual social online, (3) conducta sexual solitaria, (4) gasto sexual y (5) percepción de la gravedad del comportamiento online (que sustituye al que Delmonico definió como el interés en la conducta sexual online). 
Tecno-adicción al sexo en la población juvenil: propuesta del diseño de una escala de detección temprana

- Instrumento creado por Goodson, McCormick y Evans (2000), que estudia las actitudes y comportamientos de los estudiantes universitarios cuando usaban Internet para tres funciones principales: (1) Obtener información relacionada con la sexualidad (para la escuela, proyectos relacionados con el trabajo o información personal), (2) establecer y mantener relaciones (como usar el correo electrónico o participar en grupos de chats), y (3) la gratificación sexual (excitación sexual y/o entretenimiento).

- El inventario de uso de la ciberpornografía -Cyber-Pornography Use Inventory(CPUI) (Grubbs, Sessoms, Wheeler, y Volk, 2010) consta de 31 ítems. Se tomaron ítems del ISST, y algunos se modificaron para el uso de pornografía online en lugar del comportamiento sexual general online. EI ISST original estaba dirigido a la compulsividad sexual y la adicción online en general, y el CPUI se diseñó específicamente para áreas de la pornografía en Internet. Sus ítems se relacionan con otras escalas: escala de Compulsividad y escala Social; también se relacionaron con la subescala de aislado y la subescala de interés. Adicionalmente, se consideran otros ítems que evalúan la culpabilidad (niveles de incomodidad y auto-reproche que podrían estar asociados al uso de pornografía en Internet, en especial dentro de una población religiosa) por el uso de pornografía y un esfuerzo de evaluación invertido en la obtención de pornografía (evalúa los esfuerzos exorbitantes que realizan para obtener una sustancia o como respuesta a un comportamiento adictivo). Sus tres factores principales son: (1) Patrones adictivos, (2) culpabilidad, y (3) comportamiento sexual online-social.

- El Cuestionario de Experiencia Sexual Online -Online Sexual Experience Questionnaire-(Shaughnessy, Byers, y Walsh, 2011) consta de 9 ítems que evalúan: (1) La experiencia de las actividades sexuales online (OSA) sin excitación de los sujetos participantes, (2) la excitación-solitaria, (3) la experiencia de las actividades sexuales online (OSA) asociada. Los participantes también informaron sobre la frecuencia con la que se habían involucrado en cada comportamiento durante el último mes.

- La Prueba de Adicción a Internet-Sexo -Internet Addiction Test-Sex (IATsex) (Brand, Laier, Pawlikowski, Schchtle, Schöler, y Altstötter-Gleich, 2011), se trata de una versión modificada del IAT (Test de adicción a Internet), donde los términos "online" o Internet se sustituye respectivamente por "actividad sexual online" y "sitios de Internet por sexo". Esta versión del test de 20 ítems evalúa: (1) las quejas subjetivas de la vida cotidiana debido a las actividades sexuales online, y (2) los posibles síntomas de la adicción al cibersexo.

- La escala de uso de Internet para propósitos sexuales -Modificada -Internet Usage Scale for Sexual Purposes -Modified- (Velezmoro, Negy, y Livia, 2012) es 
Tecno-adicción al sexo en la población juvenil: propuesta del diseño de una escala de detección temprana

una versión modificada de la encuesta de Goodson et al. (2000) que consta de 25 ítems que evalúan: (1) Ver Material Sexualmente Explícito (SEM), (2) buscar compañeros sexuales, (3) buscar información relacionada con el sexo.

- Debido al componente histórico en la relación entre el sexo y la religión, se ha creado un instrumento para evaluar el uso de la pornografía en Internet que podría resultar especialmente eficaz en poblaciones religiosas (Joshua B. Grubbs, John Sessoms, Dana M. Wheeler, y Fred Volk, 2010). Se trata del Inventario de Uso de Pornografía Cibernética -Cyber Pornography Use Inventory-(CPUI) de Grubbs, Sessoms, Wheeler, y Volk, (2010) de 32 ítems. Este instrumento de tres factores: (1) Patrones adictivos (compuesto por ítems de la escala de esfuerzos, escala de culpa, escala de aislado, y escala de esfuerzos). (2) Culpabilidad (compuesto por elementos de la subescala original de culpa y de la escala original de compulsividad). Y (3) Comportamiento Sexual Online-Social -Online Sexual Behavior-Social- como aparece en e ISST (compuesto por elementos de la escala de esfuerzos preconcebidos que finalmente se descarta, y de la escala de comportamiento sexual online-social del ISST original). También analiza los sentimientos de culpa y angustia como resultado de un uso de la pornografía por Internet.

- Algunos de los mismos autores creadores del inventario anterior CPUI concretamente Joshua B. Grubbs, Fred Volk, Julie J. Exline, Kenneth I. Pargament, (2015), tratan de acortar y refinar la CPUI para explorar las asociaciones entre la adicción percibida a la pornografía en Internet y el funcionamiento psicológico, con el CPUI-9. También se explora el uso problemático de la pornografía en Internet, la hipersexualidad y la adicción a la pornografía en Internet, y la adicción percibida a la pornografía por Internet a través de las "diferencias individuales". Esta nueva medida es una medida corta de 9 ítems de la adicción percibida a la pornografía en Internet que examina su relación con varias medidas de angustia psicológica. Analiza principalmente la adicción percibida y uso, la adicción percibida y diferencias individuales, y la adicción percibida y angustia psicológica. Los tres factores fueron: (1) La compulsividad percibida, (2) los esfuerzos de acceso, y (3) la angustia emocional. Este trabajo demostró que la relación entre la angustia psicológica y la nueva medida persistió aún cuando fueron controlados otros factores potenciales como el neuroticismo, el autocontrol, y la cantidad de tiempo dedicado a ver pornografía. Las medidas que utiliza para la creación de esta herramienta son el CPUI completo más ítems que evalúan el uso de la pornografía en Internet, por un lado; y medidas de neuroticismo, autocontrol, deseabilidad social, trastorno psicológico, depresión, ansiedad, y estrés como "diferencias individuales", por otro. En definitiva, la escala demostró fuertes correlaciones con tendencias hipersexuales, reforzando una comprensión del uso compulsivo de la pornografía como un subdominio de la hipersexualidad. Los diversos componentes del CPUI-9 se asociaron con la angustia psicológica, y esto se refuerza con la idea de que la angustia psicológica está más 
Tecno-adicción al sexo en la población juvenil: propuesta del diseño de una escala de detección temprana

significativamente relacionada que con los niveles reales de uso (Joshua B. Grubbs, Fred Volk, Julie J. Exline, Kenneth I. Pargament, 2015).

\subsection{Escalas previas seleccionadas en función de los factores}

Con respecto a las escalas previas a destacar en relación a los ítems que se crearán en un futuro decir, que las escalas previas que se han considerado han sido las referidas al sexo a través de Internet. También para la construcción de la escala que se propone en este trabajo de investigación, se han estudiado las escalas referidas a Internet y a la compulsividad, aunque definitivamente la construcción de esta escala se ha realizado principalmente con las escalas relativas al trastorno sexual, a los trastornos de compulsividad y a otros trastornos adyacentes y declarados en el DSM. Por lo que la intención es la de hacer una valoración de trastornos independientes que a su vez explique la comorbilidad y el origen del comportamiento. Asimismo, dichos componentes adyacentes deben ser capaces de percibir un cierto riesgo de comportamiento para cada uno de los trastornos adyacentes; es decir, será capaz de predecir un riesgo de comportamiento agresivo, un riesgo de comportamiento antisocial, un riesgo de depresión, riesgo de ansiedad, un riesgo de comportamiento obsesivo; en definitiva, un riesgo de patología que se libera al realizar la acción "buscar sexo por Internet", lo que supondría una relación directa entre el sujeto estudiado y los trastornos adyacentes, capaces de hacer su propia valoración independiente a través de indicios que explican dichos comportamientos adyacentes. En otras palabras, será posible detectar en el individuo estudiado indicios de depresión, de agresividad, de fobia social, de ansiedad, estrés, y así cada uno de los factores que compone la escala propuesta.

A través de las investigaciones previas se obtiene información sobre las múltiples posibilidades de ver el mismo fenómeno en las que se apoyará la construcción de esta escala futura. Este modelo considera al fenómeno como un trastorno sexual en Internet, donde Internet es un medio necesario para que se pueda llevar a cabo la conducta trastornada ya que posee unas características propias y únicas capaces de impulsar comportamientos, incluido el sexual; así como ayudar a la retro-alimentación de trastornos, incluido el sexual; y donde cuya plataforma ha conseguido crear nuevas formas de sexualidad y por tanto, nuevos trastornos sexuales de orden tecnológico a partir de los trastornos sexuales ya existentes. En definitiva, el individuo puede llegar a un trastorno sexual en Internet debido al uso de la tecnología, lo que configura un nuevo fenómeno o trastorno sexual; por ello este modelo también se apoya en ver al fenómeno como un trastorno del comportamiento sexual que hay que valorar como tal, que puede encontrar explicación a través de trastornos adyacentes reconocidos en el DSM-5, y donde además, a través de los estudios anteriores, dichos factores (trastornos adyacentes) han mostrado parecer tener peso significativo suficiente para ser asociados a esta problemática. Así, debido a la propia estructura de ítems independientes adyacentes emocionales, es posible predecir que la escala que se propondrá en un futuro deberá ser capaz de detectar la situación emocional del individuo en ese momento.

En esta línea, se consideran las siguientes escalas previas relativas a la construcción 
Tecno-adicción al sexo en la población juvenil: propuesta del diseño de una escala de detección temprana

de la escala que se propondrá en un futuro, y que se dividen en dos partes diferenciadas:

\section{Parte A: Trastornos sexuales}

1. Escalas referentes a los trastornos sexuales.

2. Escalas referentes a los trastornos sexuales compulsivos.

3. Escalas referentes al comportamiento sexual en niños.

4. Escalas referentes a los síntomas sexuales.

\section{Parte B: Trastornos adyacentes}

- Escalas referentes al trastorno de depresión.

- Escalas referentes al trastorno de ansiedad.

- Escalas referentes al trastorno de agresividad, y violencia.

- Escalas referentes al trastorno de déficit de atención e hiperactividad (TDAH).

- Escalas referentes a trastornos relativos con el entorno social o ansiedad social, tales como la fobia social e integración social.

- Escalas referentes a trastornos relativos con la obsesión, a la compulsión y a la impulsividad, incluidas referencias del trastorno obsesivo-compulsivo (TOC).

\section{Parte A: Trastornos sexuales}

1. Escalas referentes a los trastornos sexuales. Se destaca la escala referente al uso problemático de la pornografía con el objetivo de evaluar el uso de la pornografía en general sin especificar el uso tecnológico para poder observar los comportamiento problemáticos de base en el uso de pornografía en general. En este orden se hace referencia a la siguiente escala:

- Escala de Uso Problemático de Pornografía -Problematic Pornography Use Scale(PPUS) cuyo desarrollo psicométrico ha sido realizado por Ariel Kor, Sigal ZilchaMano, Yehuda A. Fogel, Mario Mikulincer, Rory C. Reid, Marc N. Potenza (2014). A pesar de que esta escala no contempla el uso tecnológico, vamos a analizarla dando la importancia que merece a la otra parte del trastorno; es decir, a la parte de los trastornos sexuales destacando el uso de la pornografía, ya que coincide con el planteamiento de la escala que en este trabajo se propone al referirnos por "buscar sexo por Internet", en una primera parte, a la búsqueda de fotos o vídeos eróticos o pornográficos. EI PPUS consta de 12 ítems que evalúan 4 factores: (1) Problemas de angustia, (2) uso excesivo, (3) dificultades de control, y (4) uso para escalar/evitar emociones negativas. Los resultados informan que las puntuaciones del PPUS se correlacionan positivamente con medidas de psicopatología, por lo que se debería explorar como una adicción conductual. Esto apoya la teoría de la implicación de procesos psicológicos en la formación de este tipo de adicciones. Además, también se correlacionó positivamente con la baja autoestima y el poco apego o un vínculo pobre. Los resultados también informan que aunque las 
Tecno-adicción al sexo en la población juvenil: propuesta del diseño de una escala de detección temprana

puntuaciones del PPUS se relacionan con otras adicciones comportamentales se han distinguido claramente de las características de las adicciones conductuales relacionadas con el juego y el uso de Internet. De esta manera, es posible pensar que debido a que ambos trastornos (en este caso, el uso problemático de Internet como trastorno y el uso problemático de pornografía como trastorno) comparten características y por ello es fácil la sensación de mezcla de conceptos; sin embargo, al igual que algunas características pueden coincidir lo que hace posible relacionarse, otras características parece ser que no coinciden por lo que ahí se encuentran las diferencias que las define como otro fenómeno. El trabajo de estos autores puede prever la importancia de considerar las diferencias entre trastornos al igual que las similitudes. Quizá por ello, puede resultar conveniente revisar también los trastornos por separado como se propone al inicio de este estudio.

2. Escalas referentes a los trastornos sexuales compulsivos. Debido a la importancia de la compulsividad sexual para este tipo de trastornos sexuales y a que la compulsividad se comparte con el uso tecnológico, destacamos la siguiente escala tomada como referencia para la futura construcción de la escala que se propondrá en próximas investigaciones:

- Escala de Compulsividad Sexual -Sexual Compulsivity Scale- (SCS) que consta de 10 ítems que explican dos factores: (1) La interferencia de la conducta sexual y (2) la falta de control de impulsos sexuales (Rafael Ballester Arnal, Sandra Gómez Martínez, Ma Dolores Gil Llario, Pedro Salmerón Sánchez, 2012). Esta escala puede ser relacionada con la que se propondrá en futuras investigaciones ya que se expresa en términos de pensamientos, sentimientos, y comportamientos. Como podemos observar por ejemplo, entre otros, en el ítem 2 de dicha escala: "Mis pensamientos y comportamientos sexuales están causando problemas en mi vida" y en el (ítem 7) "Siento que los pensamientos y sentimientos sexuales son más fuertes que yo".

3. Escalas referentes al comportamiento sexual en niños. Con el objetivo de conocer el comportamiento sexual en niños se toma como referencia la siguiente escala:

- Inventario del Comportamiento Sexual en Niños -Child Sexual Behavior Inventory(CSBI). Este inventario de 38 ítems está diseñado para obtener información sobre los comportamientos sexuales en niños así como comprender el comportamiento sexual normativo de la niñez (William n. Friedrich, Jennifer Fisher, Daniel Broughton, Margaret Houston, y Cosntance R. Shafran, 1998). El CSBI contiene nueve factores que se corresponden también con el comportamiento en adultos: (1) Frontera de problemas, (2) Exhibicionismo, (3) Comportamiento del rol de género, (4) Autoestimulación, (5) Ansiedad sexual, (6) Interés sexual, (7) Intrusión sexual, (8) Conocimiento sexual, y (9) Conducta voyeurista. (William n. Friedrich, Jennifer Fisher, Daniel Broughton, Margaret Houston, y Cosntance R. Shafran, 1998). Según estos autores, el comportamiento sexual en niños se relacionó con la edad del niño, la educación materna, la sexualidad familiar, la violencia y estrés familiar. Las conductas sexuales en niños pueden ser expresadas a través de un curso evolutivo 
Tecno-adicción al sexo en la población juvenil: propuesta del diseño de una escala de detección temprana

en frecuencias de 2 a 5 años, de 6 a 9 años, y de 10-12 años, donde parece que las primeras conductas sexuales en los niños provienen de la imitación de conductas sexuales que han visto demostrar o han oído hablar. También nos informan que existe una relación entre las variables familiares y los comportamientos sexuales; y además parece darse una asociación entre los comportamientos sexuales y problemas generales de conducta. Este inventario está diseñado para que sea administrado a los padres.

4. Escalas referentes a los síntomas sexuales. Con respecto a los síntomas se toma como referencia la siguiente escala:

- Escala de Evaluación de los Síntomas Sexuales -Sexual Sympton Assessment Scale- (SSAS). Escala adaptada por Raymond, N. Et al. (2007). Esta escala se refiere principalmente a los comportamientos sexuales problemáticos, y hace referencia a los impulsos en participar en conductas sexuales problemáticas, a la frecuencia y el tiempo dedicado, a la preocupación por participar, también se refiere al control de impulsos, y a los pensamientos en participar, así como el control de los pensamientos en participar; además, explica la tensión y excitación anticipada a participar, la excitación y placer que sitió el sujeto al participar, la angustia emocional provocada, y los problemas personales (en las relaciones, problemas legales, de trabajo, y de salud) derivados de un comportamiento sexual problemático.

\section{Parte B: Trastornos adyacentes}

5. Escalas referentes al trastorno de depresión. Las escalas que se revisan con el objetivo de evaluar la tristeza, la infelicidad, el sentimiento de incomprensión por parte de los demás, y la falta de cariño, son las siguientes:

- Inventario de Depresión de Beck (1978) -Beck Depression Inventory- (BDI) que consta de 21 ítems que evalúa el componente cognitivo de la depresión tal como la desesperanza, la falta de concentración, falta de autoestima, entre otros. También es capaz de medir síntomas depresivos conductuales, afectivos y fisiológicos. Está diseñada para la administración en la población adulta, aunque también existe otra versión para la población infantil (kovacs y Beck, 1977).

- Escala de Desesperanza de Beck (1974) consta de 20 ítems para autoadministración que identifica el trastorno psicótico con riesgo de suicidio.

- Cuestionario de Pensamientos Automáticos -Automatic Thoughts Questionnaire (ATQ-30) realizada por Hollon SD y Kendall PC (1980) que consta de 30 ítems que se relacionan con el pensamiento negativo asociado a la depresión que aparece de manera automática.

Ninguna de estas escalas incluían ítems que hicieran referencia al uso tecnológico ni al comportamiento que de tal uso se deriva relacionado con los trastornos depresivos.

6. Escalas referentes al trastorno de ansiedad. Las escalas que se revisan con el 
Tecno-adicción al sexo en la población juvenil: propuesta del diseño de una escala de detección temprana

objetivo de evaluar la inquietud, estrés, la inseguridad o falta de confianza, angustia, y estar alterado que incluye lo relativo a la tensión psicológica, que deriva en un uso tecnológico, son las siguientes:

- Escala de Ansiedad para Niños de Spence-Versión en español -Spanish versión of The Spence Children's Anxiety Scale- de 38 ítems que representan 6 factores: (1) Ataque de pánico/Agorafobia, (2) Ansiedad de Separación, (3) Fobia Social, (4) Miedo al Daño Físico, (5) Trastorno Obsesivo Compulsivo, y (6) Ansiedad Generalizada, de donde Laura Hernández-Guzmán, Graciela Bermúdez-Ornelas, Susan H. Spence, Manuel Jorge González Montesinos, José I. Martínez-Guerrero, Javier Aguilar Villalobos, y Julia Gallegos Guajardo (2010) consideran que sólo el trastorno de ansiedad de separación es el único factor propio de la niñez y adolescencia; y también apuntan que la clasificación realizada se mantiene en el DSM-IV-TR.

- Inventario de Ansiedad Estado-Rasgo -State-Trait Anxiety Inventory (STAI) para la población general que costa de 40 ítems que soportan 2 factores: (1) AnsiedadEstado, y (2) Ansiedad-Rasgo a través de ítems formados con frases básicas que se utilizan normalmente para la autodescripción de sí mismo.

Ninguna de estas escalas incluían ítems que hicieran referencia al uso tecnológico ni al comportamiento que de tal uso se deriva relacionado con la ansiedad y la ansiedad sexual.

7. Escalas referentes al trastorno de agresividad, hostilidad y violencia. Las escalas que se revisan para evaluar el enfado y la rabia, donde se incluyen los celos; y que se eligen por ser considerados instrumentos de evaluación para la personalidad y sus trastornos, son las siguientes:

- Agreessión Quetionnaire-Refined versión -Cuestionario de Agresión (AQ)- Versión refinada- que David Gallardo-Pujol, Uwe Kramp, Carlos García- Forero, Meritxell Rérez-Ramírez, Antonio Andrés-Pueyo, (2006) adaptan para la población española. Este cuestionario está basado sobre el Buss- Durkee Hostility Inventory (BDHI) Inventario de Hostilidad de Buss- Durkee, que según David Gallardo-Pujol, Uwe Kramp, Carlos García-Forero, Meritxell Rérez-Ramírez, Antonio Andrés-Pueyo, (2006); y Buss AH y Perry M. (1992) lo redefinen para mejorar sus propiedades psicométricas, y el resultado fue el Cuestionario de Agresión (AQ). La adaptación española del $A Q$ versión refinada es una escala corta que consta de 12 ítems que miden cuatro aspectos de la agresión: (1) Agresión Física y (2) Agresión Verbal, que implican herir o dañar a otros, representan el componente instrumental o motor del comportamiento; (3) Hostilidad, que consiste en sentimientos de mala voluntad e injusticia, representa el componente cognitivo del comportamiento, además en la hostilidad también se incluyen los celos; y (4) Enfado, que implica la excitación fisiológica y la preparación para la agresión (en nuestro caso se prepara para la acción que es buscar sexo por Internet), representa el componente emocional o afectivo del comportamiento. Además, también este cuestionario ha sido usado para investigar la idea suicida y trastornos de la alimentación. 
Tecno-adicción al sexo en la población juvenil: propuesta del diseño de una escala de detección temprana

- También se revisa otra versión del Cuestionario de Agresión (AQ) que es el cuestionario breve de agresión de 29 ítems que aparece en el trabajo de Gregory D. Webster; C. Nathan DeWall; Richard S. Pond Jr.; Timothy Deckman; Peter K. Jonason; Bonnie M. Le; Austin Lee Nichols; Tatiana Orozco Schember; Laura C. Crysel; Benjamin S. Crosier; C. Veronica Smith; E. Layne Paddock, John B. Nezlek; Lee A. Kirkpatrick; Angela D. Bryan; Renée J. Bator, 2013) y que evalúan los mismos cuatro factores anteriores de (1) Agresión Física, (2) Agresión Verbal, (3) Enfado, y (4) Hostilidad.

- Del Inventario de Hostilidad de Buss-Durkee (BDHI) se extrae el (ítem 4) "Me enfurezco con facilidad pero se me pasa rápido", que coincide con el (ítem 7) "... me enfado rápidamente aunque se me pasa deprisa”, de la Cuestionario de agresión -versión refinada y adaptada al español de David Gallardo-Pujol, Uwe Kramp, Carlos García-Forero, Meritxell Rérez-Ramírez, Antonio Andrés-Pueyo, (2006). En ambos casos es posible observar como de alguna manera puede denotar un carácter cambiante y de arrebato ante las definiciones sobre el enfado "se me pasa rápido" o "se me pasa deprisa".

Ninguna de estas escalas tenían ítems que hicieran referencia al uso tecnológico relacionado con la agresividad, la violencia, y la hostilidad.

8. Escalas referentes al trastorno de déficit de atención e hiperactividad (TDAH). Las escalas que se revisan para evaluar la dificultad, la inquietud, la distracción, y la dificultad para concentrarse (distraerse con facilidad), evitar tareas difíciles, y no finalizar tareas escolares, son:

- Adult ADHD Self-Report Scale-V1.1 (ASRS-V1.1) Symptoms Checklist -Escala autoinforme de comprobación de síntomas de TDAH en adultos-V1.1- (World Health Organization, 2003). Esta escala está diseñada para que las preguntas sean contestadas a través de la simulación de un diálogo entre el paciente y el experto que consiste en 18 criterios del DSM-IV-TR. Sus 18 ítems se dividen en 2 partes, siendo la parte primera (parte A) la que consideran que contiene los ítems base para el cribado del ASRS-V1.1., y hace referencia al déficit de atención, en lo que se refiere a la actividad ante la dificultad. Por otro lado, La parte B, explica la hiperactividad. En definitiva, esta escala contiene ítems que evalúan (1) el déficit de atención y (2) la hiperactividad. En general, de esta herramienta, se valora la dificultad, la dificultad para mantener la atención, la dificultad para concentrarse, el ser distraído, y el sentirse inquieto.

- Escala SNAP IV adaptada a la Argentina para la evaluación del déficit de atención e hiperactividad (Nora Grañana, Alba Richaudeau, Carlos Robles Gorriti, Martín O'Flaherty, María Elena Scotti, Lucila Sixto, Ricardo Allegri y Natalio Fejerman, 2011) que consta de 20 ítems para evaluar niños y adolescentes que está diseñada para ser administrado a padres y maestros. Los ítems se dividen en dos dominios: (1) Déficit de atención e (2) hiperactividad-impulsividad, que se dividen en tres subtipos de TDAH: (a) con predominio de déficit de atención; (b) con predominio de hiperactividad-impulsividad; y (c) mixto. 
Tecno-adicción al sexo en la población juvenil: propuesta del diseño de una escala de detección temprana

Ninguna de estas escalas incluían ítems que hicieran referencia al uso tecnológico ni al comportamiento que de tal uso se deriva relacionado con el déficit de atención e hiperactividad.

9. Escalas referentes a trastornos relativos con el entorno social o ansiedad social, tales como la fobia social e integración social. Para poder detectar problemas relacionados con la fobia social se recurre a las siguientes escalas:

- Test Escala de Ansiedad Social de Liebowitz (LSAS) -Liebowitz Social Anxiety Scale Test- (R. G. Heimburg y R.E. Becker, 2002). Está escala ha sido desarrollada por el psiquiatra e investigador Dr. Michael R. Liebowitz para evaluar la forma en que la fobia social desempeña un papel en la vida de las personas a través de una variedad de situaciones. Esta escala consta de 24 ítems que responden a dos preguntas expresadas por miedo y evitación, y es para ser administrada a la población en general. De la escala LSAS destacamos la observación por la que el sujeto puede provocar la apertura de otras vías de comunicación como puede ocurrir con Internet, donde se crean otras identidades y el anonimato parece servir para la adquisición de habilidades sociales.

- Cuestionario de ansiedad social para niños (CASO-N24) desarrollado por Vicente E. Caballo, Benito Arias, Isabel C. Salazar, Marta Calderero, María J. Irurtia y Thomas H. Ollendick (2012). Este cuestionario mide la ansiedad social en niños de 9 a 15 años, y consta de 24 ítems y replica prácticamente por completo la estructura factorial del cuestionario anterior para adultos, hallando un factor más. Los 6 factores son: (1) Interacción con el sexo opuesto, (2) Hablar en público/Interacción con profesores, (3) Quedar en evidencia/Hacer el ridículo, (4) Interacción con desconocidos, (5) Expresión de molestia, desagrado o enfado, y (6) Actuar en público.

Ninguna de estas escalas tenían ítems que hicieran referencia al uso tecnológico relacionado con la fobia social, ansiedad social, comportamiento anti-social, o la interacción social.

10. Escalas referentes a trastornos relativos con la obsesión, a la compulsión y a la impulsividad, incluidas referencias del trastorno obsesivo-compulsivo (TOC). Este trastorno se considera a partir de cuando resulta difícil controlar los pensamientos. Con objetivo de detectar el pensamiento recurrente que puede convertirse en obsesivo, y finalmente en compulsividad relacionado con el sexo y el uso tecnológico se destacan las siguientes escalas que trabajan con el trastorno obsesivo-compulsivo (TOC):

- Subescala OCS-CBCL de Nelson (Nelson et al., 2001), para la evaluación del trastorno obsesivo-compulsivo en la población infanto-juvenil que analiza su validez en una muestra española Santiago Batlle, Lurdes Duñó, Ester Camprodon, Xavier Estrada, Marta Aceña, Elena Pujals, y Luis Miguel Martín (2013). Según Santiago Batlle et al. (2013). La principal característica del trastorno TOC es la presencia recurrente de obsesiones y compulsiones que interfieren en la vida cotidiana de la persona. Esta escala está creada a partir de la $\mathrm{CBCl}$ como herramienta de 
Tecno-adicción al sexo en la población juvenil: propuesta del diseño de una escala de detección temprana

detección de los trastornos incluidos en el DSM (Santiago Batlle, Lurdes Duñó, Ester Camprodon, Xavier Estrada, Marta Aceña, Elena Pujals, y Luis Miguel Martín, 2013; Achenbach y Dumenci, 2001; Achenbach y Rescorla, 2001; Achenbach y Rescorla, 2007). Siguiendo esta línea, investigadores del departamento de psiquiatría de la universidad de Washington han desarrollado una subescala del CBCL para el cribado del TOC en la infancia y adolescencia (OCS-CBCL) (Santiago Batlle, Lurdes Duñó, Ester Camprodon, Xavier Estrada, Marta Aceña, Elena Pujals, y Luis Miguel Martín, 2013; Nelson, Hanna, Hudziak, Botteron, Heath y Todd, 2001). La OCS-CBCL está diseñada para ser administrada a los padres, pero también hay versiones para los profesores y de autoaplicado para los propios jóvenes. La OCSCBCL es una escala corta de 8 ítems.

- The Obsessive Compulsive Inventory -revised (OCl-R) de Foa et al. (2002). El Inventario Obsesivo Compulsivo- Revisado consta de 18 ítems que se refieren a las experiencias que muchas personas tienen en su vida cotidiana.

No se han encontrado ítems en ninguna de estas escalas que hicieran referencia al uso tecnológico ni al comportamiento que de tal uso se deriva relacionado con el trastorno obsesivo-compulsivo.

La impulsividad se representa a través de las siguientes escalas, con el objetivo de encontrar la relación entre dichas escalas con el ítem que corresponderá en nuestra futura escala para evaluar la impulsividad. Los comportamientos impulsivos han sido definidos como comportamientos no premeditados y explosivos, y la impulsividad era la tendencia a presentar comportamientos inesperados e irreflexivos. A este concepto se añade después, además de la irresistibilidad, el deseo intenso y repetitivo de realizar un acto incluso cuando éste tiene consecuencias negativas (Lilian Salvo G. y Andrea Castro S., 2013; Moeller F, Barratt E, Dougherty D, Schimtz J, Swann A., 2011). Se plantea que la impulsividad es un constructo multidimensional, que está formado por rasgos que incluyen la tendencia a tomar decisiones de una manera rápida, la tendencia a actuar sin pensar y la tendencia a involucrarse en conductas riesgosas (Lilian Salvo G. y Andrea Castro S., 2013; Stanford M, Mathias C, Dougherty D, Lake S, Anderson N, Patton J., 2009; Squillace M, Picón J, Schmidt V, 2011; Steinberg L, Sharp C, Stanford M, Tharp A., 2013). La mayoría de las veces, el individuo percibe una creciente tensión o excitación antes de cometer la acción o acto, y como consecuencia al cometer dicho acto el individuo recibe experiencias placenteras, de gratificación o de alivio. Tras el acto puede haber o no arrepentimiento, autoreproches o culpa (Lilian Salvo G. y Andrea Castro S., 2013; American Psychiatric Association, 2000). La impulsividad forma parte de distintos trastornos, representando un criterio diagnóstico o un síntoma significativo (Lilian Salvo G. y Andrea Castro S., 2013). Adicionalmente, la impulsividad se ha relacionado principalmente con la conducta suicida (Lilian Salvo G. y Andrea Castro S., 2013; Bobes J, Sáiz P. García-Portillo M, Bascarán M, Bousoño M., 2004; Nock M., 2009), y con la agresividad (Lilian Salvo G. y Andrea Castro S., 2013; Stanford M, Mathias C, Dougherty D, Lake S, Anderson N, Patton J., 2009; MartínezTenorio F, Hernández-Daza M, Chávez-Dueñas M, 2007; Arias N, Ostrosky-Solís F., 2008), aunque también se la correlacionado con múltiples desordenes impulsivos como las auto-agresiones, búsqueda de sensaciones, consumo de alcohol y drogas, trastorno 
Tecno-adicción al sexo en la población juvenil: propuesta del diseño de una escala de detección temprana

bipolar, déficit de atención, trastorno de la conducta alimentaria, trastorno de personalidad límite y trastorno de la personalidad antisocial (Lilian Salvo G. y Andrea Castro S., 2013; Stanford M, Mathias C, Dougherty D, Lake S, Anderson N, Patton J., 2009; Steinberg L, Sharp C, Stanford M, Tharp A., 2013; Patton J, Stanford M, Barratt E, 1995; Andreu J, Fernández M, Penado M, 2012; Sierra P, Livianos L, Peris L, Rojo L, 2011). Lo que explica la relación de retroalimentación entre trastornos (adyacentes), y la estrecha asociación con los trastornos (factores). Estas escalas son las siguientes:

- Escala de impulsividad de Barratt (BIS-11) que consta de 30 ítems y que Lilian Salvo G. y Andrea Castro S. (2013) emplean en adolescentes, está formada por tres subescalas: (1) impulsividad cognitiva, (2) impulsividad motora, y impulsividad no planeada.

- Escala de Impulsividad de Plutchik (EI), que Miguel Á. Alcázar-Córcoles, Antonio J. Verdejo, José C. Bouso-Sáiz (2015) han validado para la población adolescente hispanohablante. Estos autores nos informan sobre las altas correlaciones positivas con la escala de riesgo de violencia, búsqueda de sensaciones y con las dimensiones de personalidad del cuestionario EPQ (antisocial, psicoticismo, sinceridad y neuroticismo). La impulsividad es uno de los factores más importantes que explican la conducta violenta y otras conductas como la hiperactividad o la conducta adictiva (Miguel Á. Alcázar-Córcoles, Antonio J. Verdejo, José C. BousoSáiz, 2015). Esta investigación aporta que hay menores puntuaciones de impulsividad si la agresión es hacia las personas que hacia las cosas, y en este sentido parece ser que Internet a conseguido cosificar al individuo así como convertirse en un objeto de adquisición, por lo que es posible que el "efecto pantalla" sea capaz de apoyar esta línea para poder considerar que la agresividad es mayor a través de Internet que en las relaciones reales "cara a cara". Otro hallazgo sorprendente fue que las chicas mostraron igual impulsividad que los chicos, pero sin embargo, las chicas mostraron significativamente menores puntuaciones en todos los componentes de búsqueda de sensaciones, excepto en la búsqueda de experiencias (Miguel Á. Alcázar-Córcoles, Antonio J. Verdejo, José C. Bouso-Sáiz, 2015). La escala El consta de 15 ítems

En general, ninguna de las escalas revisadas hacen referencia al uso tecnológico o de Internet, y ello es debido a que se han seguido los criterios de los trastornos que se ordenan en el DSM para la selección de escalas que se emplearan en la construcción de la escala que se propone en este trabajo.

\section{CONCLUSIONES}

Cada vez más personas utilizan Internet para actividades sexuales online y su consumo está aumentando rápidamente (Stefano Eleuteri, Francesca Tripodi, Irene Petruccelli, Roberta Rossi, Chiara Simonelli, 2014; Döring, 2009), de ahí la preocupación de la comunidad científica. También, la comunidad de salud mental ha sido testigo del dramático aumento en el uso problemático de la pornografía en Internet (Joshua B. Grubbs, Fred Volk, Julie J. Exline, Kenneth I. Pargament, 2015; Carroll et al., 2008; Manning, 2006; Owens, Behun, Manning, y Reid, 2012). 
Tecno-adicción al sexo en la población juvenil: propuesta del diseño de una escala de detección temprana

La tarea de evaluar este fenómeno resulta ser una tarea difícil. Muchos de estos trabajos científicos que se han ocupado de las nuevas tecnológicas de la Información y Comunicación (TIC), donde se han interesado en crear herramientas que puedan ser capaces de medir para detectar si un usuario es adicto al teléfono móvil (como instrumento anterior al teléfono inteligente, que en aquellos años generó unas nuevas aportaciones de portabilidad y perpetuidad; otras escalas se han ocupado en detectar si el usuario es adicto a Internet, adicto al Smartphone, adicto al sexo por Internet, etc. De esta manera, una gran parte de la investigación se ha centrado en el dispositivo (teléfono móvil, Smarphone, y ordenadores); otros muchos trabajos se han interesado en Internet (como plataforma tecnológica y fuente de la actividad); algunos otros estudios se han dirigido a partir de los contenidos o actividad (juegos, sexo, compras, etc.); otros se han centrado en los valores de la adicción; otros se han guiado por los criterios diagnósticos del DSM; otros se centran en los síntomas; y otros también se han acercado a través de los tratamientos. Asimismo, se ha reconocido en muchos de estos trabajos, que los factores emocionales cobran mayor importancia en los últimos años al tratar de comprender el fenómeno, por lo que parece ser que es óptimo considerar también los factores psico-sociales en la evaluación de la problemática. Al igual parece necesario ahondar en otros trastornos como la depresión, la ansiedad, la agresividad y la violencia, el déficit de atención e hiperactividad (TDAH), trastornos sexuales, y trastornos de integridad social u otros; a fin de encontrar los motivos que originan el comportamiento problemático, a la vez que explican la comorbilidad. La investigación científica también ha trabajado principalmente sobre adicción al cibersexo, adicción a la pornografía en Internet. Hay trabajos que se refieren a la ciber-pornografía; y otros hacen referencia a la exposición de imágenes sexualmente explícitas o a material sexualmente explícito (SEM). Sin embargo, debido al creciente número de posibilidades para obtener sexo por Internet, ha provocado que además de a la pornografía y al cibersexo, también se les preste atención a otras actividades sexuales que ocurren en Internet, considerando que la actividad sexual online viene acompañada de problemas sexuales online (OSP) (Stefano Eleuteri et al., (2014).

Este artículo relaciona la búsqueda de sexo por Internet con los conceptos de trastornos sexuales o lo que según el DSM-5 actualmente se consideran disfunciones sexuales, trastornos parafílicos, y disforia de género, dado en los conceptos principalmente de parafílias (fantasías) como búsqueda de nuevas sensaciones, y masturbación como consecución última de placer y satisfacción al terminar de realizar la acción o el comportamiento susceptible de ser compulsivo y convertirse en patológico, a través de la "búsqueda de fotos o vídeos eróticos o chats de sexo".

El modelo que aquí se propone no dejando de considerar la compulsividad a partir de un impedimento para hacer otras cosas y como perturbación de la vida diaria, que al mismo tiempo también hace referencia a la percepción del comportamiento; también, a través de la falta de control de impulsos o impulsividad por la que el individuo trata dejar de buscar sexo por Internet pero no lo consigue en firme, se expresa la incapacidad para controlar la conducta; además también se trabaja con el pensamiento obsesivo que el individuo ha interiorizado y por el que cuando algo le recuerda el sexo siente impulsos de buscar sexo por Internet, siguiendo el modelo del pensamiento recurrente del trastorno obsesivo-compulsivo (TOC), recogiendo de esta manera elementos psiquiátricos. 
Tecno-adicción al sexo en la población juvenil: propuesta del diseño de una escala de detección temprana

Adicionalmente, pretende también trabajar con factores causales que expliquen el origen del comportamiento problemático a través de elementos psicológicos y sociales como la angustia, la depresión, el estrés, la autoestima, la agresividad, el aburrimiento, el uso lúdico o por divertimento, la soledad y la falta de amigos como componente social, ante la dificultad o falta de interés en representación del trastorno por déficit de atención e hiperactividad (TDAH), que a su vez representa al posible componente genético, considerando estos factores como retroalimentadores del comportamiento adictivo. Con respecto a buscar sexo por Internet cuando el individuo quiere divertirse y pasar un buen rato puede apoyar la idea que explique que la expectativa de resultado de creencias positivas al participar en un comportamiento van a menudo formando las primeras etapas de la adicción (Daniel L. King, Paul H. Delfabbro, Mark D. Griffiths, y Michael Gradisar, 2012; Kouimtsidis, Reynolds, Drummond, Davis, y Tarrier, 2007), por lo que el cuestionario que se propondrá en un futuro podría detectar las primeras fases de la adicción para su detección precoz. En referencia a buscar sexo por Internet me impide hacer otras cosas que querría hacer ,a través de Daniel L. King, Paul H. Delfabbro, Mark D. Griffiths, y Michael Gradisar (2012) es posible explicar que en este caso la nueva información y la recompensa de buscar sexo por Internet que retroalimenta el comportamiento se basa en la gratificación de necesidades. Estas necesidades no satisfechas previas del individuo pueden ser satisfechas a través de otro camino que termine por convertirse en un aislamiento y exclusión debido a la preferencia de acción del sujeto, en este caso virtual.

La mayoría de las investigaciones han tratado de evaluar el fenómeno a través de pasos hacia el exterior (del individuo); sin embargo, esta investigación trata de caminar hacia el interior (del sujeto) de alguna manera. A diferencia de otros autores y otras investigaciones, esta investigación busca el origen causal del comportamiento, donde dicho comportamiento llevará al individuo a un uso causal de la pornografía en Internet y a la búsqueda de sexo por Internet. Este trabajo se interesa por las emociones, sentimientos, pensamientos y creencias, apoyándose sobre la premisa que la unión del uso de la tecnología, principalmente Internet, y la juventud ha provocado un cierto desorden entre los conceptos amor y sexo, donde el amor parece confundirse con el sexo, mezclándose así lo sexual con el amor y el afecto de sentirse querido y valorado. De esta manera, parece que actualmente se está configurando un nuevo modelo de las relaciones entre los jóvenes, que se apoya en el uso de la tecnología para tener razón de ser. De igual manera, se puede entender que Internet abre el mundo de la diversidad y las posibilidades pero siempre a favor de un rendimiento. En definitiva, parece que se está formando una visión desvirtualizada del amor entre los más jóvenes debido al uso de la tecnología y los conceptos erróneos de la sexualidad.

También, se ha observado que el uso de la tecnología dependiente de Internet para ciertas actividades como redes sociales, chats, etc., parecen estar sirviendo a la vez para la vigilancia y el control entre y de los "usuarios" por lo que es posible pensar que debería existir un saneamiento del uso tecnológico en general que permita la libertad digital y tecnológica de los individuos.

Este trabajo de investigación se centra principalmente en bases psicológicas, psicosociales y psiquiátricas que encuentran parámetros para conseguir detectar el origen causal de los comportamientos sexuales problemáticos básicos en Internet.

Lo que se pretende es conseguir en el futuro una escala que no haga alguna 
Tecno-adicción al sexo en la población juvenil: propuesta del diseño de una escala de detección temprana

referencia sexual explícita en alguno de sus ítems, ya que el objetivo es que pueda ser administrada también a menores de edad; y que en este sentido, permita contestar a los participantes con un mayor grado de libertad y sin algún sentimiento de vergüenza o culpa, así como por decoro no despertar ninguna figura sexual en sus pensamientos que no existiera con anterioridad a la administración de dicho test.

Esta patología ha sido asociada a la hipersexualidad, a un comportamiento compulsivo sobre Internet, a un comportamiento compulsivo-obsesivo sobre el sexo, entre otros. Sin embargo, este trabajo defiende, en parte, la tesis que podría tratarse de alguna manera de un TOC, debido a las relaciones o asociaciones directas. También el modelo que aquí se propone entiende que los trastornos de adicción al sexo por Internet se consideran un subtipo de la adicción al sexo. Además, se considera que los trastornos hipersexuales son un subtipo del trastorno obsesivo-compulsivo (TOC), y esto es debido a la relación directa entre hipersexualidad y compulsividad, una falta de control de impulsos y un pensamiento obsesivo recurrente. En este sentido, se trata de integrar el componente de TOC o comportamiento obsesivo unido a unas determinadas emociones, estímulos y respuestas que el sujete es capaz de realizar.

Otros autores han considerado el análisis de la angustia psicológica o angustia emocional. Sin embargo, los ítems que se proponen en una escala futura están basados en el estudio (y existencia) de las diferentes emociones y no en un único concepto de angustia psicológica o emocional. $Y$ donde además dichas emociones están adicionalmente relacionadas al ser consideradas como trastornos debido a que son factores que están extraídos de lo que el DSM-5 define como trastorno.

El estudio de las emociones o estados emocionales del individuo a través de una escala o test hace posible que aún prescindiendo del elemento compulsividad, sea posible detectar una problemática o patología de este tipo. De esta manera, se podría obtener una escala futura que tenga una doble medición: por un lado, detectaría la problemática a través de las emociones del sujeto; y por otro, a partir de elementos de compulsividad y/o del comportamiento obsesivo, así como de la impulsividad.

En definitiva, parece ser que las escalas extraídas a partir de los modelos que sostienen las herramientas de medición previas que se ofrecen en este artículo son óptimas para evaluar el fenómeno de la tecno-adicción al sexo en una población infantil, adolescente y juvenil.

Adicionalmente, la escala futura pretende además referenciar y confirmar la importancia de los estímulos percibidos, tanto en contenido como en forma.

También, de igual manera, la escala futura está construida para poder observar la importancia de la imagen y el poder que ejerce sobre los individuos dicha forma y contenido.

La escala futura que se creará desde el modelo propuesto, pretende tratar de recoger información sobre la naturaleza del problema, ya que es muy difícil obtener información, incluso en la clínica, sobre la sexualidad de los más jóvenes en Internet y de sus experiencias a través de la red. Además, también se intenta concienciar a través de la administración del cuestionario a los jóvenes como experiencia de aprendizaje.

A través de la escala que se creará en un futuro también plantea poder reconocer la posibilidad de predecir entornos problemáticos del individuo, los cuales pueden ser relacionados directamente con un uso problemático y patológico de las nuevas 
Tecno-adicción al sexo en la población juvenil: propuesta del diseño de una escala de detección temprana

Tecnologías de la Información y Comunicación.

\section{FUTURAS INVESTIGACIONES}

Como futuras investigaciones se plantea la creación de la escala así como la presentación de la misma.

\section{BIBLIOGRAFIA}

Achenbach, T.M., y Dumenci, L. (2001). Advances in Empirically Based Assessment: Revised Cross-Infor- mant Syndromes and New DSM-Oriented Scales for the CBCL, YSR, and TRF: Comment on Lengua, Sa- dowksi, Friedrich, y Fisher. Journal of Consulting and Clinical Psychology, 69, 699-702.

Achenbach, T.M., y Rescorla L.A. (2001). Manual for the ASEBA School-Age Forms \& Profiles. Burlington, VT: University of Vermont, Research Center for Children, Youth, and Families.

Achenbach, T.M., y Rescorla, L.A. (2007). Multicultural Supplement to the Manual for the ASEBA School-Age Forms \& Profiles. Burlington, VT: University of Ver- mont, Research Center for Children, Youth \& Families.

Albright, J. M. (2008). Sex in America online: An exploration of sex, marital status, and sexual identity in Internet sex seeking and its impacts. The Journal of Sex Research, 45, 175-186.

Alcázar-Córcoles, M. Á.; Verdejo, A. J. y Bouso-Sáiz, José C. (2015). Propiedades psicométricas de la escala de impulsividad de Plutchik en una muestra de jóvenes hispanohablantes. Actas Españolas de Psiquiatría, 43(5), 161-9.

Penado Abilleira, M. (2012). Agresividad Reactiva y proactiva en adolescentes: efecto de los factores individuales y socio-contextuales. Tesis doctoral. Facultad de Psicología. Departamento de Personalidad, Evaluación y Tratamiento Psicológico I (Personalidad, Evaluación y Psicología Clínica). Universidad Complutense de Madrid.

Arias García, N.; Ostrosky Solís, F. (2008). Neuropsicología de la violencia y sus clasificaciones. Revista Neuropsicología, Neuropsiquiatría y Neurociencias, 8(1), 95-114.

Ballester Arnal, Rafael; Gil Llario, Ma Dolores; Gómez Martínez, Sandra y Gil Juliá, Beatriz (2010). Propiedades psicométricas de un instrumento de evaluación de la adicción al cibersexo. Psicothema, 22(4), 1048-1053.

Ballester Arnal, R.; Gómez Martínez, S.; Gil Llario, M. D. y Salmerón Sánchez, P. (2012). Sexual Compulsivity Scale: Adaptation and Validation in Spanish Population. Routledge Taylor \& Francis Group, Journal of Sex \& Marital Therapy, $0,1-14$.

Batlle, S.; Duñó, L.; Camprodon, E.; Estrada, X.; Aceña, M.; Pujals, E. y Martín, L. M. (2013). Subescala OCS-CBCL de Nelson para la Evaluación del Trastorno Obsesivo-Compulsivo Infanto-juvenil: Análisis de Validez en una Muestra Española. Revista de Psicopatología y Psicología Clínica, 18 (1), 81- 92. 
Tecno-adicción al sexo en la población juvenil: propuesta del diseño de una escala de detección temprana

Beck, AT; Weissman, A; Lester, D. y Trexler, L. (1974). The measurement of pessimism: The hopelessness Scale. Journal of consulting and clinical psychology , 42(6), 861-865.

Block JJ. (2008). Issues for DSM-V: Internet Addiction. American Journal of Psychiatry, 165 (3), 306-307.

Bobes J, Sáiz P, García-Portillo M, Bascarán M, Bousoño M. (2004). Comportamientos suicidas. Prevención y tratamiento. Barcelona: Ars Médica.

Boies, S. C., Cooper, A., \& Osborne, C. S. (2004). Variations in Internet-related problems and psychosocial functioning in online sexual activities: Implications for social and sexual development of young adults. CyberPsychology \& Behavior, 7 , 207-230.

Brand, M., Laier, C., Pawlikowski, M., Schchtle, U., Schöler, T., \& Altstötter-Gleich, C. (2011). Watching pornographic pictures on the Internet: Role of sexual arousal ratings and psychological-psychiatric symptoms for using Internet sex sites excessively. Cyberpsychology, Behavior, and Social Networking, 14, 371-377.

Buss, A. H., \& Perry, M. P. (1992). The aggression questionnaire. Journal of Personality and Social Psychology, 63, 452-459.

Caballo, Vicente E.; Arias, Benito; Salazar, Isabel C.; Calderero, Marta; Irurtia, María J. y Ollendick, Thomas H. (2012). Un Nueva Medida de Autoinforme para Evaluar la Ansiedad/Fobia Social en Niños: El "Cuestionario de Ansiedad Social para Niños" (CASO-N24). Behavioral Psychology / Psicología Conductual, 20(3), 485-504.

Carroll JS, Padilla-Walker LM, Nelson LJ, Olson CD, Barry CM, Madsen SD. (2008). Generation XXX: Pornography acceptance and use among emerging adults. Journal of Adolescent Research, 23, 6-30.

Cooper, A., Griffin-Shelley, E., Delmonico, D. L. \& Mathy, R. (2001). Online sexual problems: Assessment and predictive variables. Sexual Addiction and Compulsivity, 8, $265-283$.

Cooper, A., Månnson, S. A., Daneback, K., Tikkanen, R., \& Ross, M. W. (2003). Internet sexuality in Scandinavia. Sexual and Relationship Therapy, 18, 277-291.

Cooper, A., Putnam, D.E., Planchon, L. S., Boies, S.C. (1999). Online sexual compulsivity: Getting tangled in the net. Sexual Addiction \& Compulsivity, 6, 79104.

Cooper, A., Scherer, C., \& Mathy, R. M. (2004). Overcoming methodological concerns in the investigation of online sexual activities. CyberPsychology \& Behavior, 4, 437447.

Corley, M. D., \& Hook, J. N. (2012). Women, female sex and love addicts, and use of the Internet. Sexual Addiction \& Compulsivity, 19, 53-76.

Daneback, K., Cooper, A., \& Månsson, S. A. (2005). An Internet study of cybersex participants. Archives of Sexual Behavior, 34, 321-328.

Daneback, K., Månsson, S. A., \& Ross, M. W. (2007). Using the Internet to find offline sex partners. CyberPsychology \& Behavior, 10, 100-107.

Delmonico, D. (1997). Internet Sex Screening Test. Recuperado de http:// www.sexhelp.com

Delmonico, D. L., \& Miller, J. A. (2003). The Internet Sex Screening Test: A comparison of sexual compulsives versus non-sexual compulsives. Sexual and Relationship Therapy, 18, 261-276. 
Tecno-adicción al sexo en la población juvenil: propuesta del diseño de una escala de detección temprana

Delmonico, D.L., Griffin, E. J. (2008). Cybersex and the e-teen: What marriage and family therapists should know. Journal of Marital and Family Therapy, 34(4), 431444.

Döring, N. M. (2009). The Internet's impact on sexuality: A critical review of 15 years of research. Computers in Human Behavior, 25, 1089-1101.

Eleuteri, S.; Tripodi, F.; Petruccelli, I.; Rossi, R. y Simonelli, C. (2014). Questionnaires and scales for the evaluation of the online sexual activities: A review of 20 years of research. Cyberpsychology: Journal of Psychosocial Research on Cyberspace, 8 (1), art. 2. doi: 10.5817/CP2014-1-2.

Foa, E.B., Huppert, J.D., Leiberg, S., Hajcak, G., Langner, R., et al. (2002). The Obsessive-Compulsive Inventory: Development and validation of a short versión. Psychological Assessment, 14, 485-496.

Friedrich, William N.; Fisher, Jennifer; Broughton, Daniel; Houston, Margaret y Shafran, Constance R. (1998). Normative Sexual Behavior in Children: A Comtemporary Sample. Pediatrics, 101(4), e9.

Gallardo-Pujol, D.; Kramp, U.; García-Forero, C.; Pérez-Ramírez, M. y Andrés-Pueyo, A. (2006). Assessing aggressiveness quickly and efficiently: the Spanish adaptation of Aggression Questionnaire-Refined versión. Elsevier, ScienceDirect, European Psychiatry, Eurpsy- 2418. http://france.elsevier.com/direct/EURPSY

Goodson, P., McCormick, D., \& Evans, A. (2000). Sex and the Internet: A survey instrument to assess college students' behavior and attitudes. CyberPsychology \& Behavior, 3, 129-149.

Grañana, N.; Richaudeau, A.; Robles Gorriti, C.; O’Flaherty, M.; Scotti, M. E.; Sixto, L.; Allegri, R. y Fejerman, N. (2011). Evaluación de déficit de atención con hiperactividad: la escala SNAP IV adaptada a la Argentina. Revista Panamericana de Salud Pública, 29(5), 344-349.

Grov, C., Gillespie, B. J., Royce, T., \& Lever, J. (2011). Perceived consequences of casual online sexual activities on heterosexual relationship: A U.S. online survey. Archives of Sexual Behavior, 40, 429-439.

Grubbs, Joshua B.; Sessoms, John; Wheeler, Dana M.; Volk, Fred (2010). The CyberPornography Use Inventory: The Development of a New Assessment Instrument. Sexual Addiction \& Compulsivity, 17, 106-126.

Grubbs, Joshua B.; Volk, Fred; Exline, Julie J. y Pargament, Kenneth I. (2015). Internet Pornography Use: Perceived Addiction, Psychological Distress, and the Validation of a Brief Measure. Journal of Sex \& Marital Therapy, 41(1), 83-106.

Heimberg, R. G., \& Becker, R. E. (2002). Cognitive-behavioral group therapy for social phobia: Basic mechanisms and clinical strategies. New York, NY: Guilford Press.

Hernández-Guzmán, L.; Bermúdez-Ornelas, G.; Spence, S. H.; González Montesinos, M. J.; Martínez-Guerrero, J. I.; Aguilar Villalobos, J. y Gallegos Guajardo, J. (2010). Versión en español de la Escala de Ansiedad para Niños de Spence (SCAS). Revista Latinoamericana de Psicología, 42 (1), 13-24.

Hollon, SD y Kendall, PC. (1980). Cognitive self-statement in depression: Development of an automatic thoughts questionnaire. Cogn Ther Res, 4, 383-395. 
Tecno-adicción al sexo en la población juvenil: propuesta del diseño de una escala de detección temprana

Kafka, M. P. (2010). Hypersexual disorder: A proposed diagnosis for DSM-V. Archives of Sexual Behavior. Advance online publication. Archives of Sexual Behavior 39(2), 377-400. Doi: 10.1007/s10508-009-9574-7

Kaplan MS, Krueger RB (2010). Diagnosis, Assessment, and Treatment of Hypersexuality: Annual Review of Sex Research, Journal of Sex Research, 47(23), 181-198.

King, Daniel L., Delfabbro, Paul H., Griffiths, Mark D. y Gradisar, Michael (2011). Assessing clinical trials of Internet addiction treatment: A systematic review and CONSORT evaluation., Clinical Psychology Review, 31, 1110-1116.

King, Daniel L., Delfabbro, Paul H., Griffiths, Mark D. y Gradisar, Michael (2012). Cognitive-Behavioral Approaches to Outpatient Treatment of Internet Addiction in Children and Adolescents. Journal of Clinical Psychology: In Session, 68 (11), 1185- 1195.

Ko, Chih-Hung; Yen, Ju-Yu; Chen, Cheng-Sheng; Yeh, Yi-Chun y Yen, Cheng-Fang (2009). Predictive Values of Psychiatric Symptoms for Internet Addiction in Adolescents.A 2-Year Prospective Study. Archives of Pediatrics\& Adolescent Medicine 163(10), 937- 943.

Ko, Chih-Hung; Yen, Ju-Yu; Liu, Shu-Chun; Huang, Chi-Fen y Yen, Cheng-Fang (2008). The Associations Between Aggressive Behaviors and Internet Addiction and Online Activities in Adolescents. Journal of Adolescent Health, 44, 598- 605.

Kor, Ariel; Zilcha-Mano, Sigal; A. Fogel, Yehuda, Mikulincer, Mario; C. Reid, Rory y Potenza, Marc N. (2014). Psycometric development of the Problematic Pornography Use Scale. Addictive Behaviors, 39, 861-868.

Kouimtsidis, C., Reynolds, M., Drummond, C., Davis, P., \& Tarrier, N. (2007). Cognitivebehavioural therapy in the treatment of addiction. Chichester, UK: John Wiley \& Sons.

Lam-Figueroa, N.; Contreras-Pulache, H.; Mori-Quispe, E.; Nizama- Valladolid, M.; Gutiérrez, C.; Hinostroza-Camposanto, W.; ... Coaquira-Condori, E. (2011). Adicción a Internet: Desarrollo y validación de un instrumento en escolares adolescentes de Lima, Perú. Revista Perú de Medicina Experimental y Salud Publica, 28(3), 462-469.

Martínez-Tenorio F, Hernández-Daza M, Chávez-Dueñas M. (2007). Niveles de agresividad e impulsividad en pacientes psiquiátricos de un hospital de tercer nivel. Neurología, Neurocirugía y Psiquiatría, 40, 35-40.

Moeller F, Barratt E, Dougherty D, Schimtz J, Swann A. (2011). Psychiatric aspects of impulsivity. American Journal of Psychiatry, 158, 1783-93.

Nock, Matthew K. (2009). Why Do People Hurt Themselves? New Insights Into the Nature and Functions of Self-Injury. SAGE journals, 18(2), 78-83.

Owens, E. W.; Behun, R. J.; Manning, J. C. y Reid, R. C. (2012). The Impact of Internet Pornography on Adolescents: A Review of the Research. Sexual Addiction \& Compulsivity, 19, 99-122.

Patton JH, Stanford MS, Barratt ES. (1995). Factor structure of the Barratt impulsiveness scale. Journal of Clinical Psychology, 51(6), 768-74.

Putnam, R. (2000) Bowling Alone: The Collapse and Revival of American Community. New York: Simon and Schuster.

Raymond, N et al. (2007). The Sexual Symptom Assessment Scale (SSAS). Sexual 
Tecno-adicción al sexo en la población juvenil: propuesta del diseño de una escala de detección temprana

Addiction \& Compulsivity, 14(2), 119-129.

Ross, M. W., Daneback, K., Månsson, S-A., Tikkanen, R., \& Cooper, A. (2003). Characteristics of men and women who complete or exit from an online internet sexuality questionnaire: A study of instrument dropout biases. Journal of Sex Research, 40, 396-402.

Ross, M.W., Månsson, S-A., Daneback, K. (2012). Prevalence, severity, and correlates of problematic sexual Internet use in Swedish men and women. Archives of Sexual Behavior, 41, 459-466.

Salvo G., Lilian y Castro S., Andrea (2013). Confiabilidad y validez de la escala de impulsividad de Barratt (BIS-11) en adolescentes. Revista Chilena de NeuroPsiquiatría, 51(4), 245-254.

Sánchez Zaldívar, Silvia e Iruarrizaga Díez, Itziar (2009). Nuevas Dimensiones, Nuevas adicciones: La Adicción al Sexo en Internet. Intervención Psicosocial 18(3)

Sardinero García, E.; Pedreira Massa, J. L. y Muñiz, J. (1997). El cuestionario CBCL de Achenbach: Adaptación española y aplicaciones clínico-epidemiológicas. Recuperado de http://www.copmadrid.org/webcopm/publicaciones/clinicas/1997/Vol8/Arti3.htm

Shaughnessy, K., Byers, E. S., \& Walsh, L. (2011). Online sexual activity experience of heterosexual students: Gender similarities and differences. Archives of Sexual Behavior, 40, 419-427.

Sierra P., Livianos L., Peris L., Rojo L. (2011). Impulsividad, búsqueda de sensaciones y agresividad en pacientes bipolares tipo I y II. Revista de Psiquiatría y Salud Mental, 4(4), 195-204.

Squillace, M., Picón Janeiro, J., Schmidt, V. (2011). El concepto de Impulsividad y su ubicación en las teorías psicobiológicas de la personalidad. Revista Neuropsicologia Latinoamericana, 3(1): 8-18.

Stanford, M. S., Mathias, C. W., Dougherty, D.M., Lake, S.L., Anderson, N.E., Patton, J. H. (2009). Fifty years of the Barratt Impulsiveness Scale: An update and review. Personality and Individual Differences, 47(5), 385-395.

Steinberg, L. Sharp, C., Stanford, M. S., Tharp, A.T. (2013). New tricks for an old measure: The development of the Barratt Impulsiveness Scale-Brief (BIS-Brief). Psychological Assessment, 25(1), 216-226.

Stulhofer, A., Busko, V., y Landripet, I. (2010). Pornography, sexual socialization, and satisfaction among young men. (Croacia). Archives of Sexual Behavior, 39(1), 16878.

Tonioni, F.; D’Alessandris, L.; Lai, C.; Martinelli, D.; Corvino, S.; Vasale, M.; Fanella, F.; Aceto, P. y Bria, P. (2011). Internet addiction: hours spent online, behaviors and psychological symptoms. General Hospital Psychiatry, 34, 80-87.

Velezmoro, R.; Negy, C.; Livia, J. (2012). Online sexual activity: cross-national comparison between United States and Peruvian college students. Archives of Sexual Behavior, 41(4), 1015-25.

Webster, Gregory D.; DeWall, C. Nathan; Pond, Richard S. Jr.; Deckman, Timothy; Jonason, Peter K.; Le, Bonnie M.; ... Bator, Renée J. (2013). The Brief Aggression Questionnaire: Psychometric and Behavioral Evidence for an Efficient Measure of Trait Aggression. Aggressive Behavior, 9999, 1-20. 